\title{
DESTACADAS PERSONALIDADES DE LA DERMATOLOGÍA TROPICAL EN EL PERÚ
}

\author{
Zuño Burstein Alva',2,a
}

\begin{abstract}
RESUMEN
En este artículo se rinde homenaje a tres personalidades médicas peruanas: José Neyra Ramírez (1929-2012), Abelardo Tejada (1931-2013) y Oscar Romero Rivas (1929-2011), se destaca sus valiosas contribuciones en el campo de la Dermatología Tropical y de la Medicina Sanitaria en beneficio de la salud pública y de la docencia medica peruana. Se hace mención de la constitución de un grupo de jóvenes investigadores a los que se denominó "Los Anacoretas", quienes con el apoyo de los profesores Hugo Pesce, Enrique Encinas, Juan Francisco Valegas en Lima y Enerst Nausk y Víctor Manchego en Alemania, con la participación del gobierno peruano y de Alemania, promovieron e hicieron realidad, el proyecto de crear el primer centro universitario de investigación de medicina tropical de la costa pacífica de América Latina denominado "Instituto de Medicina Tropical" Daniel A. Carrión de la Universidad Nacional Mayor de San Marcos de Lima (IMT/UNMSM).
\end{abstract}

Palabras clave: Dermatología; Docentes médicos; Perú (fuente: DeCS BIREME).

\section{PROMINENT PERSONALITIES FROM THE TROPICAL DERMATOLOGY FIELD IN PERU}

\begin{abstract}
This article pays tribute to three Peruvian medical personalities: José Neyra Ramirez (1929-2012), Abelardo Tejada (1931-2013) and Oscar Romero Rivas (1929-2011), highlighting their valuable contributions to the field of Tropical Dermatology and Health Medicine that benefited public health and Peruvian medical education. The article mentions the formation of a group of young researchers called "The Anacoretas", including professors Hugo Pesce, Enrique Encinas, Juan Francisco Valegas in Lima; and Enerst Nausk and Victor Manchego in Germany, who, with the support of the Peruvian and German governments promoted and created the first university research center of Tropical Medicine on the Pacific Coast of Latin America called Daniel A. Carrion Tropical Medicine Institute at Universidad Nacional Mayor de San Marcos in Lima (TMI/UNMSM).
\end{abstract}

Key words: Dermatology; Faculty, medical; Peru (source: MeSH, NLM).

En este artículo se rinde homenaje a tres personalidades médicas ya desaparecidas, quienes por su valiosa trayectoria docente asistencial, su importante producción científica y sus valiosas contribuciones sanitarias en el campo de la Dermatología Tropical y de la Medicina Sanitaria, ocupan un destacado sitial en la historia de la salud pública y de la docencia médica peruana. Este grupo de investigadores médicos de vocación científica innata de servicio, tuvieron su desempeño ejemplarizador en el "Hospital 2 de Mayo de Lima", centro hospitalario (para hombres) que pertenecía a la Beneficencia Pública de Lima, antes de su pase al Ministerio de Salud y que fuera inaugurado el 28 de febrero de 1875, como uno de los centros asistenciales hospitalarios más grandes y modernos de esa época; y que durante un prolongado lapso histórico, junto con el Hospital Arzobispo Loayza (para pacientes mujeres) igual de la Beneficencia Pública de Lima, eran los únicos centros formadores de médicos y de investigación científica para la antigua y única Facultad de Medicina "San Fernando" de la Universidad Nacional Mayor de San Marcos (UNMSM) Decana de América.

Durante la década de los 50 se constituyó en el "Hospital 2 de Mayo" un grupo de estudiantes y profesionales del área médica bajo la tutoría del profesor doctor Hugo

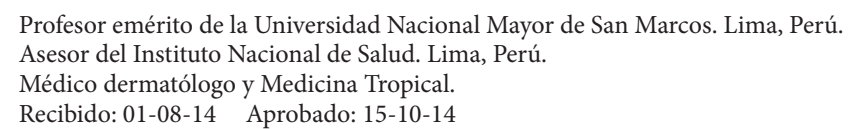


Pesce Pescetto, jefe de la cátedra de Enfermedades Infecciosas y Tropicales de la Facultad de Medicina "San Fernando" con sede en ese hospital. El Dr. Pesce, a quien hemos rendido numerosos homenajes en esta revista y en otros medios, fue un gran maestro, humanista, salubrista y leprólogo de prestigio internacional ${ }^{(1,2)}$. En este grupo se encontraba el Dr. José Neyra, colaborador del profesor Pesce en la cátedra universitaria y en la campaña antileprosa del Ministerio de Salud, así como Hugo Lumbreras, Abelardo Tejada, Óscar Romero y el suscrito; También se encontraban estudiantes y médicos de otras áreas, entre ellos: Olga Palacios, César Náquira, Humberto Guerra, Roberto Llanos, Jorge Montesinos, Francisco Morales, Mario Zubiate, Ismael Benítez, Rony Korngold, Gianfranco Piana; las biólogas Bertha Llanos, Yolanda Lisarazo, Julia Liceras (del Instituto Nacional de Salud, colaboradoras del Dr. Arístides Herrer), Carmen Villanueva, Juana Ararte y la botánica Juana Infantes, todos ellos dedicados al estudio e investigación de las enfermedades transmisibles, infecciosas, parasitarias y las causadas por animales ponzoñosos; fueron acogidos por el Prof. Dr. Enrique Encinas en su Laboratorio de patología del sistema nervioso del Hospital Psiquiátrico "Víctor Larco Herrera", con el beneplácito y apoyo de Javier Mariátegui y de su director Dr. Juan Francisco Valega, quien fue el que denominó a este grupo "Los Anacoretas".

De este grupo surgió la idea de crear un centro de investigaciones de enfermedades tropicales en el Perú, el que se materializó con el apoyo del profesor Hugo Pesce. Con el Dr. Enrique Encinas, la coordinación en Lima de Zuño Burstein, desde Alemania con Hugo Lumbreras, que había sido becado por gestión del Dr. Enrique Encinas, y con el apoyo del Dr. Víctor Manchego, agregado cultural del Perú en Alemania, y del Profesor Ernst Nauck (3) director del Tropeninstitut de Hamburgo, que vino al Perú para decidir el convenio del Gobierno Alemán y del Perú para su creación, que fue exitosa. La inauguración fue el 15 de julio de $1963^{(4)}$ con el nombre Instituto de Medicina Tropical "Daniel A. Carrión" de la UNMSM (IMT/UNMSM), el primer centro de investigación de este tipo en la costa pacífica de América Latina.

Todos los miembros constituyentes de este grupo científico denominado "Los Anacoretas" tuvieron con el tiempo destacadas posiciones profesionales, académicas y como investigadores médicos. Cabe mencionar y destacar que Hugo Lumbreras fue fundador del otro Instituto de Medicina Tropical "Alexander Von Humboldt" de la Universidad Peruana Cayetano Heredia y Humberto Guerra fue, posteriormente, director de ese centro y vicerrector de investigaciones de dicha universidad. Abelardo Tejada, Olga Palacios,
Cesar Náquira y Zuño Burstein tuvieron posteriormente responsabilidades de dirección en el IMT/UNMSM y en el INS/MINSA.

Es la década de los 60 , con ocasión y durante la severa crisis universitaria determinada por la renuncia masiva de docentes de la Facultad de Medicina de la UNMSM, por discrepancia con la política universitaria del gobierno nacional y la posterior creación de una universidad privada con los docentes renunciantes, se encomienda a un porcentaje alto de miembros del grupo de "Anacoretas" recrear las cátedra de Dermatología, abandonada por sus docentes renunciantes, y reajustar la cátedra de Medicina Tropical ${ }^{(5)}$, para lo cual se comprometió a nuevos docentes mediante concurso de méritos. El éxito de la reconstitución de la cátedra de Medicina Tropical coordinada por el Dr. José Neyra Ramírez con otros docentes, y de la cátedra de Dermatología con los Dres. Oscar Romero y Abelardo Tejada, con la coordinación del Dr. Zuño Burstein y, posteriormente, del Dr. Aizic Cotlear como profesor principal, se logró la constitución de un cuerpo docente de gran calidad, que asumió la docencia de pre y posgrado; y por disposición legal la autorización del otorgamiento a nombre de la Nación de los primeros títulos universitarios de especialidad en Medicina Tropical y Dermatología bajo la modalidad inicialmente no escolarizada y, posteriormente, escolarizada con residencias de la especialidad de gran rigurosidad ${ }^{(5)}$.

Seguidamente, una semblanza biográfica recordatoria de tres personalidades, ya desaparecidas, protagonistas de lo mencionado en párrafos anteriores.

El Dr. José Neyra Ramírez, médico-cirujano, doctor en Medicina, especialista en Medicina Tropical (Leprología) y Neumología (Tisiología), con dedicación profesional a la salud pública y a la docencia universitaria, nació en Lima el 12 de marzo de 1920, de padres arequipeños. Sus estudios médicos los realizó en la Facultad de Medicina "San Fernando" de la Universidad Nacional Mayor de San Marcos (UNMSM), perteneció a la promoción médica 1949 "Hermilio Validizán" (5). Terminó sus estudios en el tercer puesto de orden de méritos de 149 alumnos, con la tesis para obtener el grado de bachiller en Medicina titulada: "La correlación inmunológica de la lepra con la tuberculosis". Obtuvo el título de médico cirujano el año 1950; y se graduó de doctor en Medicina en 1972 con la tesis: "El Programa Nacional de Control de la Tuberculosis, su evaluación" luego obtuvo el título de especialista en Medicina Tropical en la UNMSM el año 1975.

El Dr. Neyra, becado por el gobierno francés, realizó sus estudios de posgrado en tuberculosis y tisiología infantil 


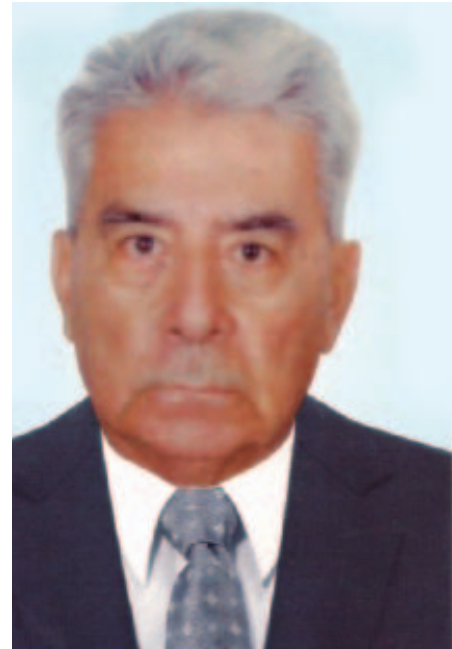

JOSÉ NEYRA RAMÍREZ (1920-2012)

de 1951 a 1953, en el Instituto Pasteur y en la Facultad de Medicina de París. Posteriormente, estudió en la misma ciudad Broncoesofagología, Neumotisiología y prevención y tratamiento de la tuberculosis infantil; el año 1962, en Brasil, hizo estudios sobre lepra, tuberculosis y BCG. En 1968 llevó el curso regular de Salud Pública en la Escuela de Salud Pública del Perú. Ha concurrido, como participante, expositor y organizador, en un gran número de certámenes científicos en el Perú y en el extranjero, sobre Neumología, tuberculosis, Salud Pública, educación médica, Dermatología y lepra. Cabe mencionar que el año 1978 fue delegado del Perú a la XXXI Asamblea Mundial de la Salud en Ginebra y delegado de la Asociación Peruana de Facultades de Medicina (ASPEFAN) para diferentes seminarios sobre regímenes académico-administrativos de las facultades de medicina, y a la XI Conferencia Panamericana de Educación Médica, en México, el año 1986.

Trabajó durante 32 años para el Ministerio de Salud del Perú, ingresó el año 1947 como ayudante en el Departamento de Lepra, se integró al grupo de leprólogos que rodeaban al Prof. Hugo Pesce y colaboró con él durante toda la vida de ese gran maestro; cumplió importante apoyo administrativo en la "Campaña Antileprosa" que logró el control sanitario de la lepra en el Perú ${ }^{(6-9)}$. También participó en la cátedra de Enfermedades Infecciosas, Tropicales y Parasitarias de la UNMSM que jefaturaba el Dr. Pesce, donde se desempeñó como coordinador docente y organizador del fichero bibliográfico de la cátedra y el de la División de Lepra del Ministerio de Salud, que cumplieron un importante papel referencial y docente para la universidad y para el Ministerio de Salud del Perú. En el Ministerio de Salud (MINSA) ascendió a médico auxiliar de la División de Lepra y sucesivamente a médico jefe de la Sección de Leprología, así como de la Sección de Organización y Control; fue, asimismo, supervisor de la Dirección de Normas y jefe del Programa de Control de Tuberculosis y Lepra. Posteriormente, se desempeñó como director general de programas de salud, inspector general de salud. El año 1978 fue nombrado viceministro de salud y, a su cese, quedó como asesor honorario de lepra y tuberculosis del MINSA.

En la Escuela de Salud Pública del MINSA se desempeñó, desde 1975, como director de coordinación educativa y en 1976 se le encargó la Dirección General de esta Escuela, formadora de médicos sanitarios y técnicos salubristas. Su experiencia médico asistencial se llevó a cabo como médico asistente del Sanatorio Olavegoya (Jauja, Perú), en 1950; en la Sala San Vicente del Hospital Dos de Mayo, de 1951 a 1954; en la Sala Santa Rosa (TBC) de dicho hospital, de 1954 a 1962 y en el Sanatorio de Guía (lepra), de 1951 a 1955.

El Dr. Neyra dedicó gran parte de su vida a la actividad docente universitaria, ingresó en 1954 como jefe de clínica en la cátedra de Enfermedades Infecciosas, Tropicales y Parasitarias, con el Dr. Hugo Pesce, obtuvo por concurso de méritos la condición de profesor auxiliar, asociado y principal, y asumió la jefatura, desde el año 1972, de la asignatura de Medicina Tropical y la presidencia del Comité de Segunda Especialización en Medicina Tropical, desde 1983 a 1989; donde se encargó de la organización, capacitación y otorgamiento del título de médico especialista en Medicina Tropical a nombre de la Nación. A su cese administrativo, después de 40 años de actividad docente, fue nombrado profesor emérito vitalicio de la UNMSM, continuando con la docencia y colaboración universitaria hasta su sensible fallecimiento.

Como colaborador en la cátedra de Medicina Tropical del Prof. Hugo Pesce (10) intervino en la reestructuración de esa cátedra durante la crisis universitaria de los años 60 y apoyó al grupo de jóvenes investigadores denominados "Anacoretas", apoyado por los Prof. Hugo Pesce y Enrique Encinas, en el propósito de crear el primer instituto de Medicina Tropical "Daniel A. Carrión", de la UNMSM. Fue Secretario docente-administrativo de la Facultad de Medicina en varios períodos y jefe de la Oficina de Internado, donde se desempeñó con éxito, responsabilidad y con la amabilidad que lo caracterizaban, gozó de gran aprecio del cuerpo docente y de los estudiantes de Medicina, quienes le dieron su nombre a la promoción médica del año 1982. Tuvo, además, una intensa actividad médico-gremial representativa, fue fundador y miembro activo de instituciones médico-científicas, sociales y de gobierno. 
Desde 1956 a 1958 ocupó el cargo de secretario de la Federación Médica Peruana; de 1969 a 1971 fue secretario del Consejo Regional III del Colegio Médico del Perú y de 1994 a 1995 decano del Colegio Médico del Perú, cargo al que fue elegido por votación médica a nivel nacional y que se vio obligado a dejar para ocupar el cargo, también elegido a nivel nacional, como primer magistrado médico del Consejo Nacional de la Magistratura, que ejerció de 1995 al 2000, organismo encargado de la vigilancia, nombramiento y remoción de jueces en todo el país.

Incorporado a la Academia Nacional de Medicina en 1985, como académico asociado, institución del más alto nivel académico científico en el Perú, creada por ley para asesorar a los poderes del Estado en problemas de salud, fue promovido a académico de número en 1993 y pasó a la condición de académico emérito el año 2000. Fue académico honorario de la Academia Peruana de Salud; miembro fundador de la Sociedad Peruana de Epidemiología, presidente fundador de la Sociedad Peruana de Enfermedades Infecciosas y Tropicales; presidente fundador de la Unión Médica Franco-Peruana; vicepresidente de la Sociedad Peruana de Salud Pública; secretario ejecutivo de la Asociación Peruana de Facultades de Medicina; secretario general de la Asociación de Médicos Cesantes del Ministerio de Salud; miembro del Consejo de Ciencias y Tecnología (CONCYTEC), entre otras instituciones médico-científicas; fue, además, nombrado teniente alcalde del Concejo Distrital de Breña, Lima, de 1979 a 1986.

Ha contribuido en la realización de numerosos trabajos de investigación de sus especialidades y publicado más de 60 trabajos sobre tuberculosis, lepra, fiebre amarilla, peste, medicina sanitaria, verruga peruana, histoplasmosis, educación médica y numerosas contribuciones sobre historia de la medicina peruana, en revistas médicas y otros órganos de difusión. Su fecunda y muy valiosa contribución a la medicina, la salud pública, la docencia y gran trabajo institucional, ha merecido un gran número de distinciones y condecoraciones, como la de "Gran Oficial de la Orden Hipólito Unanue" y de la Orden "Daniel A. Carrión" (1985) del Ministerio de Salud del Perú; Mención Honrosa Premio "Cayetano Heredia" a las mejores publicaciones médicas del año 1951; designación como miembro correspondiente de la Sociedad Francesa de Tuberculosis y del Comité de Terapéutica de la Unión Internacional contra la Tuberculosis (1961); consejero de la Unión Latinoamericana de Sociedades de Tisiología (1967); Premio "Anibal Corvetto" al mejor trabajo de Tisiología (Lima, 1966); Miembro de la Unión Internacional Contra la Tuberculosis (1971), entre otras.
El Dr. José Neyra falleció en Lima el 4 de diciembre de 2012, a los 92 años de edad, luego de un proceso neurológico con compromiso multiorgánico, de relativamente corta evolución, precipitado por el reciente y abrupto fallecimiento de su esposa de nacionalidad francesa, Marie Claude Soupplet, con quien tuvo cuatro hijos.

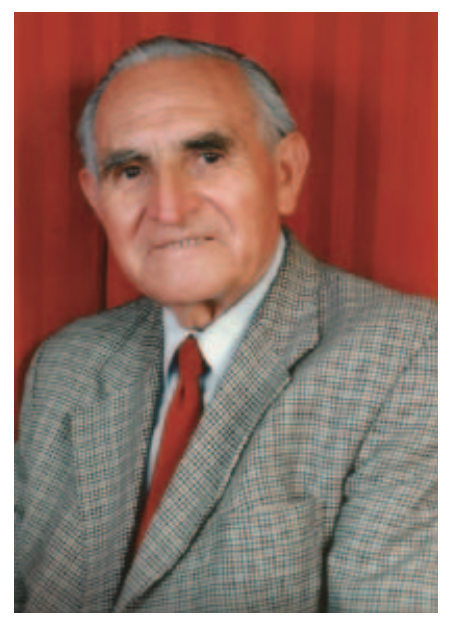

\section{ALBELARDO TEJADA VALENCIA} (1931-2013)

El Doctor Abelardo Tejada Valencia nació en Cabanillas, Puno, Perú, el año 1931; recibió el título de médicocirujano en la Facultad de Medicina de San Fernando de la Universidad Nacional Mayor de San Marcos (UNMSM), en 1962. Desde estudiante, y durante toda su vida, se interesó y trabajó intensamente en aspectos clínicos, epidemiológicos y de investigación científica de las enfermedades tropicales peruanas, teniendo una intensa y competente vocación para el trabajo de campo, en la búsqueda de reservorios y captura de vectores de las enfermedades regionales, así como en el estudio de las poblaciones nativas, que dieron motivo a numerosas publicaciones y aportes originales; conocimiento aplicado a la docencia y a la epidemiología de nuestra patología. Obtiene el grado académico de doctor en Medicina en la UNMSM, en 1974. Inicia su actividad médica como asistente muy tempranamente desde el año 1954 en el Ministerio de Salud, Subregión de Salud del Callao, en la División de Enfermedades Transmisibles, llegando a ser jefe del Programa de Enfermedades Venéreas del Callao, médico del Servicio de Sanidad Marítima y jefe del Centro de Salud del Callao.

Simultáneamente, inicia su carrera docente en la UNMSM, que desempeña por más de 50 años, iniciados el año 1954, como ayudante de cátedra 
$y$, posteriormente, como jefe de prácticas, profesor auxiliar, profesor asociado y profesor principal en la cátedra de Enfermedades Tropicales, liderada por el Dr. Hugo Pesce, colaboró con los profesores José Neyra, Hugo Lumberas y Zuño Burstein, y formé parte del grupo denominados "Los Anacoretas", que , con el apoyo de los profesores Hugo Pesce, Enrique Encinas, Juan Francisco Valega y el profesor Nauck de Hamburgo, promovieron la creación del primer centro de investigación en Medicina Tropical del país, el Instituto de Medicina Tropical "Daniel A. Carrión" de la UNMSM, fundado en $1963^{(6-11)}$, donde llegó a ser director del 2002 al 2007, después de haber pasado por diferentes responsabilidades de alto nivel.

En el Hospital Dos de Mayo, de Lima, donde tuvo su sede inicial la Cátedra de Medicina Tropical, colaboró intensamente en la docencia e investigación y formó parte del grupo de docentes que reestructuraron exitosamente, después de una severa crisis universitaria, las Cátedras de Medicina Tropical y de Dermatología ${ }^{(6)}$. Dirigió y participó activamente en estudios de investigación en enfermedades de transmisión sexual, hepatitis B, SIDA, leishmaniasis, enfermedad de Carrión, toxoplasmosis, enfermedades virales y lepra, contribuyó en la búsqueda activa de pacientes en las zonas endémicas, colaborando con el Programa de Control y Eliminación de la Lepra en el Perú con el Dr. Zuño Burstein ${ }^{(7)}$. Su interés, dedicado a la Entomología, le permitió identificar una nueva especie de vector de la enfermedad de Carrión y de la leishmaniasis, que es una Lutzomia que lleva su nombre, Lutzomia tejadai.

Hasta cerca de su fallecimiento, y ya enfermo, siguió colaborando en la investigación de plantas medicinales peruanas, en búsqueda de un tratamiento alternativo para la Leishmaniasis, trabajó en colaboración con investigadores japoneses y dirigido en el Perú por los doctores Fernando Cabieses y Zuño Burstein. Tiene más de 100 trabajos publicados y presentados en congresos sobre diferentes tópicos de patología tropical. El Dr. Tejada fallece el 2 de agosto de 2013 por una afección pulmonar que venía arrastrando después de un linfoma a células $\mathrm{T} y$, pese a ello, continuaba atendiendo pacientes en el consultorio del Instituto de Medicina Tropical, dictando clases y colaborado en la investigación científica en la que estaba comprometido y participando activamente en el Comité Directivo del IMT/ UNMSM, del cual formaba parte.

Recibió numerosas distinciones por su dedicación a la investigación científica y la docencia, como: reconocimiento como Investigador Científico por Res. Rectoral 33662, 1971, UNMSM; diploma de honor en el V Congreso Peruano de Parasitología, 2000; diploma y Medalla "Alberto Barton", del Cuerpo Médico del Hospital "Daniel A. Carrión”, Callao, Perú, 2006; premio y Medalla al Mérito Científico, UNMSM, 2008; miembro honorario de la Asociación de Parasitología del Perú. Perteneció a numerosas instituciones científicas nacionales y extranjeras, entre ellas la Sociedad Peruana de Enfermedades Infecciosas y Tropicales, la Asociación de Parasitología del Perú, la Sociedad Peruana de Dermatología, entre otras.

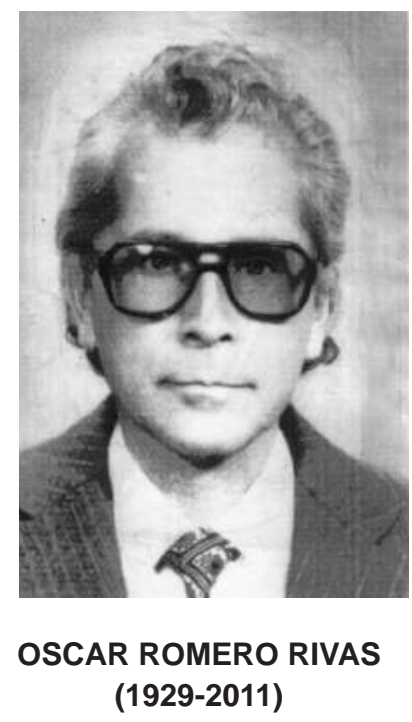

El Dr. Oscar Romero Rivas, médico dermatólogo y tropicalista, nació en Lima, Perú en 1929. Se recibió de médico-cirujano en 1961 en la Universidad Nacional Mayor de San Marcos (UNMSM) y obtuvo el título de especialista en Dermatología en la misma universidad en 1977. Hizo sus estudios especializados en la Facultad de Medicina de Sao Paulo, Brasil, y en el Hospital Das Clínicas de la misma ciudad, en Microbiología y Medicina Tropical (6). Inició su carrera docente en la Facultad de Medicina de la UNMSM en 1955, como ayudante de prácticas del Laboratorio Clínico de la Facultad en el Hospital Dos de Mayo de Lima, centro hospitalario donde permaneció toda su vida dedicado a la docencia, investigación y labor asistencial, comenzando esta última como médico venereólogo concursado, en el Centro de Salud de Ate, Vitarte, y, posteriormente, como dermatólogo concursado en el Hospital Dos de Mayo, donde llegó a ser jefe del Servicio Asistencial de Dermatología (1995-1999), completando 37 años de servicios prestados a la salud pública en el Ministerio de Salud del Perú.

Ingresó por concurso de méritos a la cátedra de Enfermedades Infecciosas, Tropicales y Parasitarias, del Prof. Hugo Pesce y, posteriormente, a la cátedra 
de Dermatología, con los profesores Aizic Cotlear y Zuño Burstein, en las que ascendió de categoría hasta llegar a ser profesor principal de ambas cátedras, completando en total 44 años de servicio docente. En la cátedra de Dermatología cumplió una función decisiva en el reflotamiento de dicha cátedra después de la crisis producida por el abandono de sus anteriores docentes, así como coordinando, en apoyo a la jefatura del Dr. José Neyra, la reestructuración de la cátedra de Medicina Tropical en ese difícil trance histórico ${ }^{(5,4)}$.

Su vocación de investigación científica se manifestó muy tempranamente, y desde sus primeros años de estudiante de Medicina inició una intensa colaboración con el Dr. Zuño Burstein, integrándose al grupo de jóvenes investigadores denominado "Los Anacoretas"; colaboró en los trabajos de campo que con el Dr. Arístides Herrer, del Instituto Nacional de Salud del MINSA, realizaban investigaciones en la búsqueda de los reservorios de los agentes causales de la enfermedad de Carrión y la leishmaniosis cutánea en las zonas endémicas del valle del Rímac, donde encontraron parásitos flagelados en plantas lactecentes de esa zona ${ }^{(13)}$, lo que replanteó la posibilidad de que estas plantas cumplieran la función de reservorios de estas enfermedades, ello dio lugar a que junto al Dr. Burstein publicaran una comunicación previa a la tesis de bachiller de este último, que estaba en preparación sobre este tópico ${ }^{(14)}$.

Con el Dr. Burstein estudió y publicó varias contribuciones científicas sobre Micología Médica, tanto sobre micosis superficiales como micosis profundas ${ }^{(15,16)}$, teniendo a su cargo el estudio de laboratorio, inicialmente, en el Laboratorio de Investigaciones Parasitológicas y Micológicas en la cátedra de Medicina Tropical y, posteriormente, en el laboratorio del Servicio Académico Asistencial de Dermatología. Este servicio académico asistencial adquirió gran prestigio y sirvió de base para la capacitación y adiestramiento, a nivel nacional, de médicos en la especialidad de Dermatología, fue donde se iniciaron los primeros residentados formales para la obtención de títulos a nombre de la nación de médicos en dicha especialidad (5) y en donde el Dr. Romero llegó a ser jefe por varios años, y profesor responsable de la especialidad y jefe de la cátedra de Dermatología de la Facultad de Medicina de la UNMSM, de 1980 a 1993.

El Dr. Romero ha publicado numerosos artículos de investigación en revistas de Dermatología y participado como ponente y organizador en certámenes nacionales e internacionales de Dermatología y Dermatología Tropical, sobre: enfermedad de Jorge Lobo (primer caso diagnosticado en el Perú), blastomicosis sudamericana; sporotrichosis; leishmaniosis cutánea; enfermedades venéreas; sífilis; donovanosis; necrobiosis lipoídica; sarcoma de Kaposi y otros temas relacionados con su especialidad. Editó, además, manuales de temas diversos para la docencia médica. En reconocimiento a sus méritos, el Dr. Romero recibió numerosas distinciones, entre las que se destaca la "Medalla de Reconocimiento por sus 25 años de Servicios al Hospital Dos de Mayo"; Medalla al Mérito por los 40 años en la docencia médica en la Facultad de Medicina de la UNMSM; distinción honorífica Medalla al Mérito por destacada labor científica y profesional, otorgada por el Colegio Médico del Perú; título honoríico de "Maestro de la Dermatología Nacional", otorgado por la Sociedad Peruana de Dermatología; Medalla al Mérito otorgada por la promoción médica "Hugo Pesce", Facultad de Medicina de la UNMSM, en 1965; colocación de nombre de "Prof. Dr. Oscar Romero Rivas" al Salón de Sesiones de profesores de la cátedra de Dermatología de la Facultad de Medicina de la UNMSM. Miembro del Colegio Ibero Americano de Dermatología (CILAD) y de la Sociedad Peruana de Dermatología. Fallece en Lima, en el 2011, después de una prolongada dolencia.

\section{REFERENCIAS BIBLIOGRÁFICAS}

1. Burstein Z. Héroes de la Salud Pública en el Perú. Hugo Pesce Pesceto. Rev Peru Med Exp Salud Publica. 2003; 20(3):172-3.

2. Burstein Z; Ministerio de Salud, Instituto Nacional de Salud. Conmemoración del XXV Aniversario de su Fallecimiento 1969-1994: Dr. Hugo Pesce Pescetto. Lima: MINSA/INS; 2014.

3. Burstein Z. Homenaje al Dr. Ernst Georg Nauck. An Fac Med. 2003;64(4):274-8.
4. Gardini W.25 Aniversario del Instituto de Medicina Tropical "Daniel A. Carrión” Revista Peruana de Medicina Tropical 1991;5:6-8.

5. Burstein Z. Historia de los programas de formación y titulación de médicos especialistas en el Perú. Rev Peru Med Exp Salud Publica. 2014;31(3):589-600.

6. Flores E. Flores L. Burstein Z. Historia de la Dermatología en el Perú. En: Galimberti R. Pierini AM Cervini AB. Historia de la Dermatología Latinoamericana. Lima: Privat Toulouse; 2007. p.307-64.
7. Burstein Z. La lepra, enfermedad paradigmática, ¿ podrá ser eliminada en un futuro cercano? Folia Dermatologica Peruana. 2002; 13 (2):51-6.

8. Neyra J. Aguilar J. Alfaro E. Epidemiologia de la lepra en el Perú. Pucallpa (Perú). Primer seminario de lepra; 1971 Set 19 25; Pucallpa: Ministerio de Salud; 1971.

9. Neyra J, Alarcón J, Piscoya J, Robles R. Evaluación operacional y epidemiologia de la Hanseniasis en el departamento de Ucayali 1981-1984. Diagnostico. 1985;4:13-7. 
10. Burstein Z. Quiebra el Programa de Control de la lepra en el Perú por la descentralización e Integración a los programas generales de salud. Arch Arg Dermatol. 1980;30:173-80.

11. Burstein Z; Ministerio de Salud; Instituto Nacional de Salud. Conmemoración del XXV aniversario de su fallecimiento 19691994: Dr. Hugo Pesce Pescetto. En: Neyra J. Hugo Pesce, el hombre, el maestro, el amigo. Lima: MINSA-INS; 2014. p. 90-5.

12. Tejada A. Historia del Instituto de Medicina Tropical «Daniel A. Carrión» de la Facultad de Medicina de San Fernando de la Universidad Nacional Mayor de San Marcos. Rev Peru Med Trop. 2004;9:101-3.

13. Burstein Z. Romero O. Flagelados en el latex de Jatropha macrantha (Huanarpo Hembra). Arch Per Pat Clin. 1956;10:1-11.

14. Burstein Z. Contribución al Estudio de la Verruga Peruana y de la UTA. Investigación en el Cnidósculosbasiacantha y Jatropha macrantha (huanarpos), como posible reservorio. Tesis de Bachiller de Medicina Facultad de Medicina. Lima: UNMSM; 1947.
15. Burstein Z. Romero R. Contribución al estudio de las micosis profundas en el Perú. Acerca de tres casos de Sporotrichosis. Rev Soc Per Derma. 1967;1:61-77.

16. Burstein Z. Romero O. Larrea P. Una forma poco usual de actinomicosis Rev Soc Per Dermatol. 1968;2(1):45-9.

Correspondencia: Zuño Burstein Alva Dirección: Cápac Yupanqui 1400. Jesús María, Perú

Teléfono: 748-111

Correo electrónico:zburstein_2000@yahoo.com

\section{Visite nuestra página en Facebook, www.facebook.com/rpmesp Infórmese sobre los eventos y los nuevos contenidos de la Revista Peruana de Medicina Experimental y Salud Pública}

\section{REVISTA PERUANA DE MEDICINA EXPERIMENTAL Y SALUD PÚBLICA} Difundiendo investigación en salud

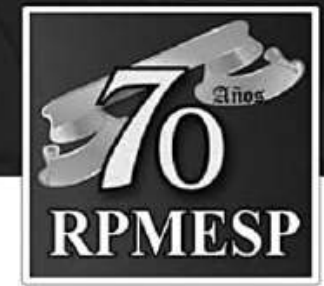
1942 - 2012
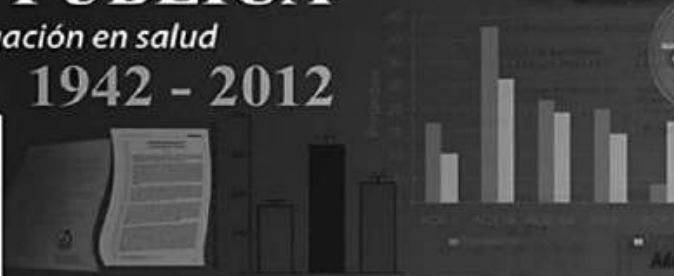

Revista Peruana de Medicina Experimental y Salud Pública (OFICIAL)

A 1254 personas les gusta esta página · 8 personas están hablando de esto

Comunidad

La Revista Peruana de Medicina Experimental y Salud Publica es el órgano oficial de difusión cientifica del Instituto Nacional de Salud

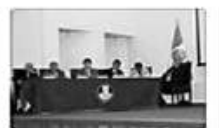

Informadón - sugerir una ecición

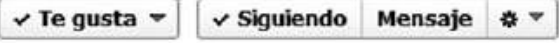

$\sim$ Te gusta
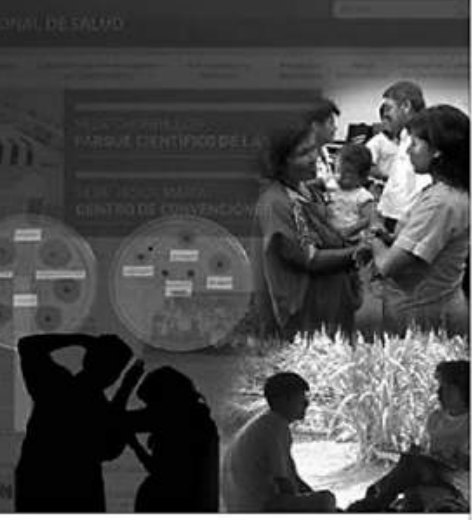